\title{
Application of FIB/SEM and TEM to Bit Failure Analyses in SRAM Arrays
}

Wentao Qin, Alex Volinsky ${ }^{1}$, Larry Rice, Lorraine Johnston and David Theodore

Digital DNA ${ }^{\mathrm{TM}}$ Labs, Motorola

MD EL622, 2100 E Elliot Road, Tempe, AZ 85284

${ }^{1}$ Department of Mechanical Engineering, University of South Florida, Tampa FL 33620

\begin{abstract}
Many microelectronic chips contain embedded memory arrays. A single SRAM bit-cell contains several transistors. Failure of any of the transistors makes the entire bit-cell inoperable. Dual-beam Focused Ion Beam (FIB) combines the slicing capability of FIB with in-situ SEM imaging. The combination offers unparalleled precision in looking for root causes of failures in microelectronic devices. Once a failure site is located, an FIB lift-off method can be used to prepare a TEM sample containing the area of interest. Further structural, elemental information can then be acquired from the failure site. We report here analyses of single and multiple bit failures in SRAM arrays carried out using FIB/SEM, and in two cases TEM imaging and EDS/PEELS. Root causes of bit failures including remnant seed-layer metal between stacked vias have been identified.
\end{abstract}

\section{INTRODUCTION}

With the advantages of high access-speed and virtually unlimited read-write endurance, SRAM has become a prevalent memory on the market. On the other hand, it is well-known to for its sensitivity to process variation. Therefore SRAM is also frequently used as a qualification vehicle for process development. As a result, failure analysis of SRAM chips is critical not only for trouble-shooting and problem-solution, but also for assurance of on-time delivery to market.

Several failure mechanisms of SRAM bits have been reported in literature, including up-shift of threshold voltage and decrease of trans-conductance of the Ld-PMOS due to local depletion in the polySi gate [1], node-to-node and node-to-power-line shorting through CMP scratches [2], electrical opens caused by dangling contacts at bitline and PMOS [3, 4], and bridging of W-plugs through W-extrusion [3]. In all of these cases the feature sizes are on the order of a tenth of a micron or larger.

In the scaling-down of microelectronics, reduction in device-sizes can increase their sensitivity to process variation. Root causes of failures can be very subtle and localized to extremely small size scales. We report here, failure analyses involving characterization of tiny features down to the size scale of 1 nm.

\section{EXPERIMENTAL DETAILS}

Failing bits were identified electrically in memory arrays, and subsequently located by a dual-beam Focused Ion Beam (FIB). In-situ SEM of the FIB was used to check for any abnormalities in the crosssection while material was being removed. In some cases SEM imaging and EDS (Energy-dispersive Spectroscopy) were sufficient to identify failure mechanisms once such abnormalities were revealed. 
However, there were occasions when the resolutions in SEM imaging and/or elemental analyses were inadequate. TEM (transmission electron microscopy) was subsequently resorted to. A TEM sample was made, by removing materials from the opposite side until the abnormal feature was exposed. FIB-SEM was then alternated from both sides until the abnormal feature was confined within a thin slice that is electron-transparent, and about $15 \mu \mathrm{m}$ long. This thin slice was lifted-off with a micro-manipulator, where it was picked up by a glass needle and placed on a thin carbon film on a $\mathrm{Cu}$ mesh.

A field-emission gun TEM/STEM (Scanning TEM) was used for brightfield imaging, as well as elemental analyses through EDS and PEELS (parallel electron energy loss spectroscopy). The elemental analyses were carried out in STEM mode with the electron beam being focused down to a "nano-probe", i.e. an electron beam of a full-width-at-half-maximum on the order of 1 nanometer.

\section{DISCUSSION}

\section{A. Failure Analyses by FIB/SEM}

Fig. 1(a) is an SEM image of via contacts from the transistor silicon to the $2^{\text {nd }}$ level of metal-line (metal2). The etch in creating the via on the left was incomplete, resulting in an absence of contact between the metal-2 and transistor. As a result, two adjacent bits were electrically open.

Fig. 1(b) is another SEM image with a field of view from the transistor up to metal-2. The metal-2 is discontinuous above a via between metal-1 and metal-2. Two bits therefore were also electrically open. The cause is a photoresist issue in patterning the metal- 2 trenches resulting in a local absence of the metal.

In addition to open bits, bits that were shorted were also analyzed. Fig. 2 is an SEM image with a field of view from the transistors to metal-2 of some shorted bits. Metal-2s in the middle are bridged together. Such a bridging was similarly caused by a photoresist issue in patterning the trenches of metal-2, and ultimately led to the shorting of the bits.
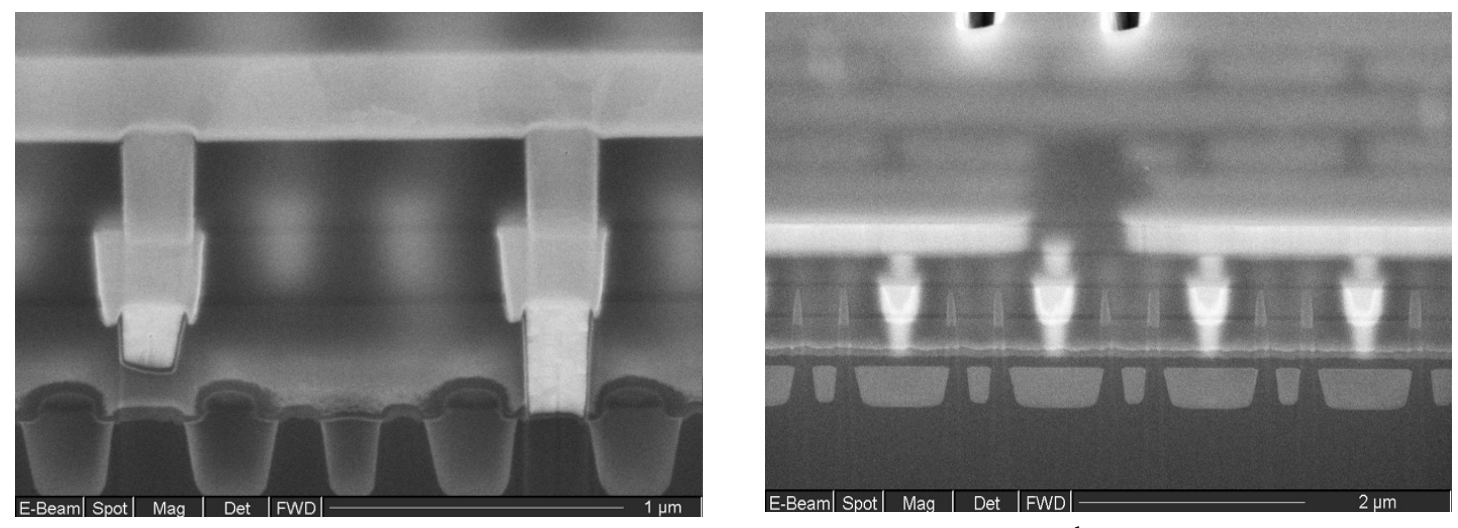

Figure 1. SEM images of via contacts from transistors to the $2^{\text {nd }}$ level of metal-lines (metal-2). As shown in Fig. 1(a), the etch to create the via on the left was incomplete, resulting in an electrical open; Fig. 1(b) reveals a discontinuity of metal- 2 above a via between metal-1 and metal-2. Two bits therefore were electrically open. 


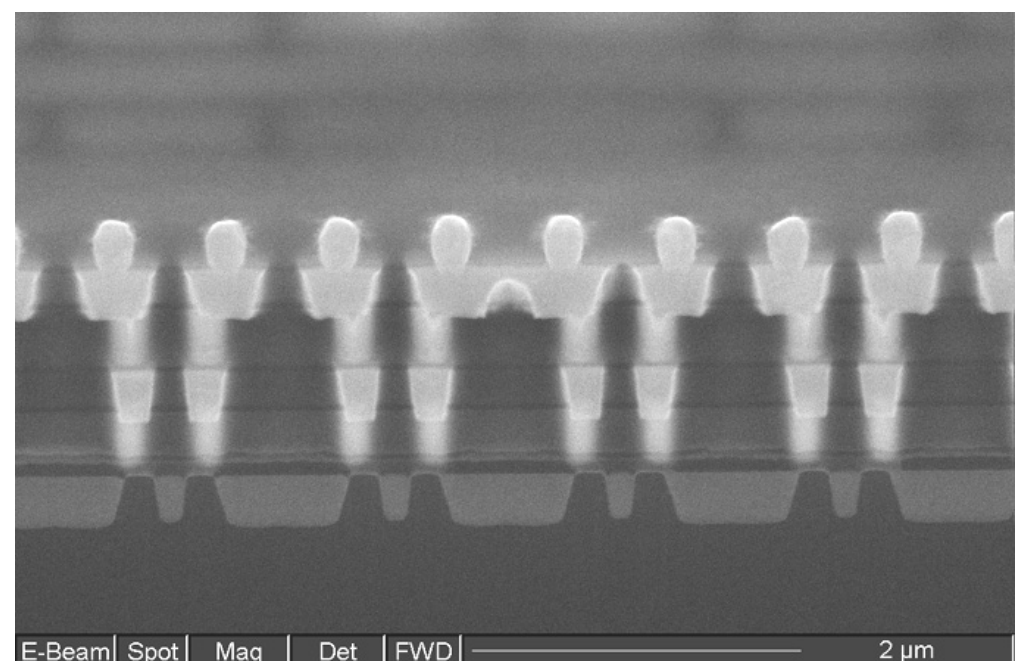

Figure 2. An SEM image with a field of view from the transistors to metal-2 of some shorted bits. Metal-2s in the middle are bridged together. Such a bridging was caused by a photoresist issue in patterning the trenches of metal-2, and is responsible for the shorting of the bits.

\section{B. Failure Analyses by FIB/SEM and TEM}

Figure 3(a) is a TEM brightfield image of a pair of tungsten-plugs of a shorted bit. There is a faint dark layer between the tungsten plugs. A higher magnification view of the faint layer is provided in Fig. 3(b). The thickness of the faint layer is measured to be $3 \mathrm{~nm}$ to $5 \mathrm{~nm}$. On FIB samples such as this one, thicknesses of re-deposition on both sides of the thin slice are comparable to that of the thin slice [5- 7]. Such a re-deposition made resolving this faint layer difficult in the brightfield image.

In order to identify the material of the layer, EDS-PEELS spectrum imaging was performed, with a nano-probe of FWHM $=1.6 \mathrm{~nm}$. A pair of EDS spectrum and PEELS spectrum were acquired at each
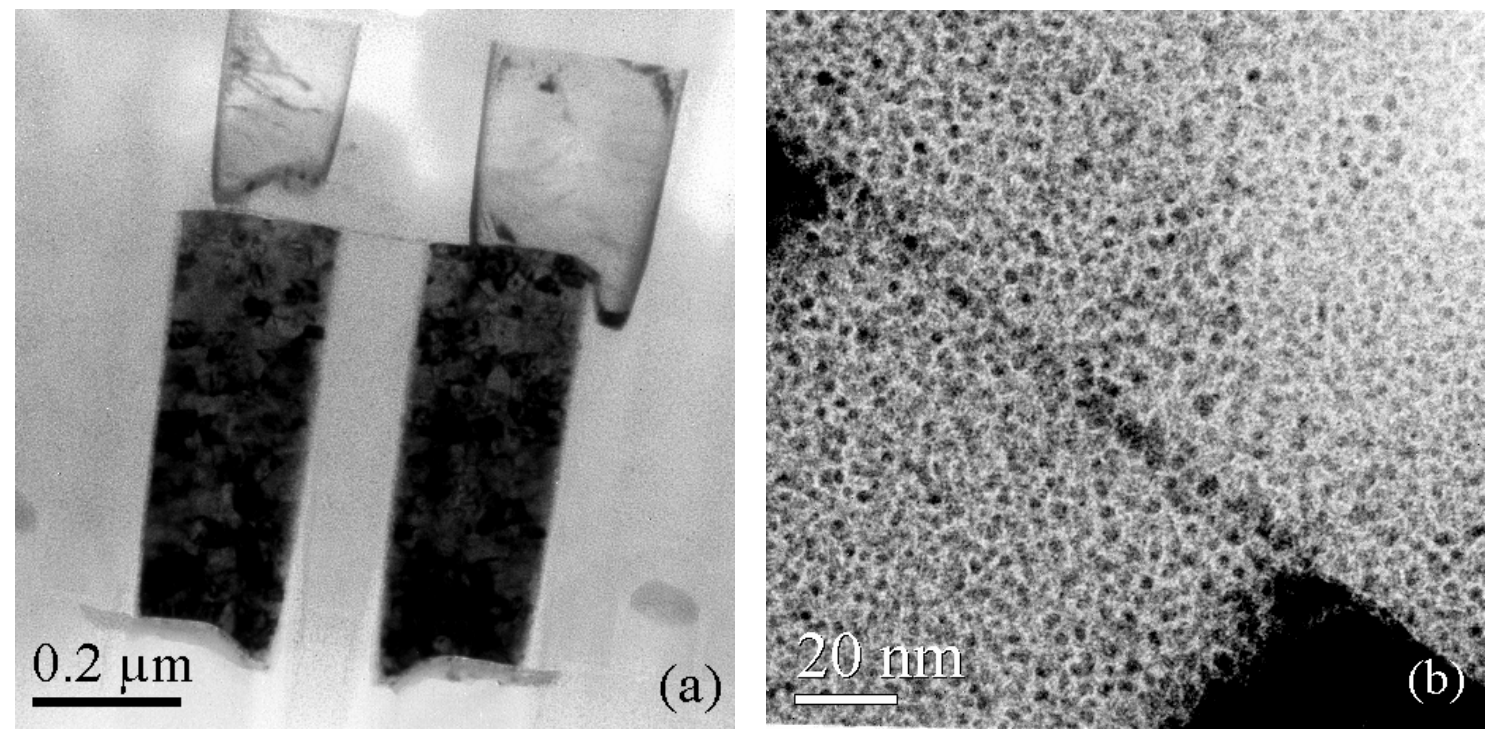

Figure 3. TEM image of a pair of tungsten-plugs of a shorted bit (a). There is a faint dark layer between the tungsten-plugs. A higher magnification view of the faint layer is provided in Fig. 3(b). The dark layer is some residual titanium that was left behind by the CMP (chemical-mechanical planarization). 

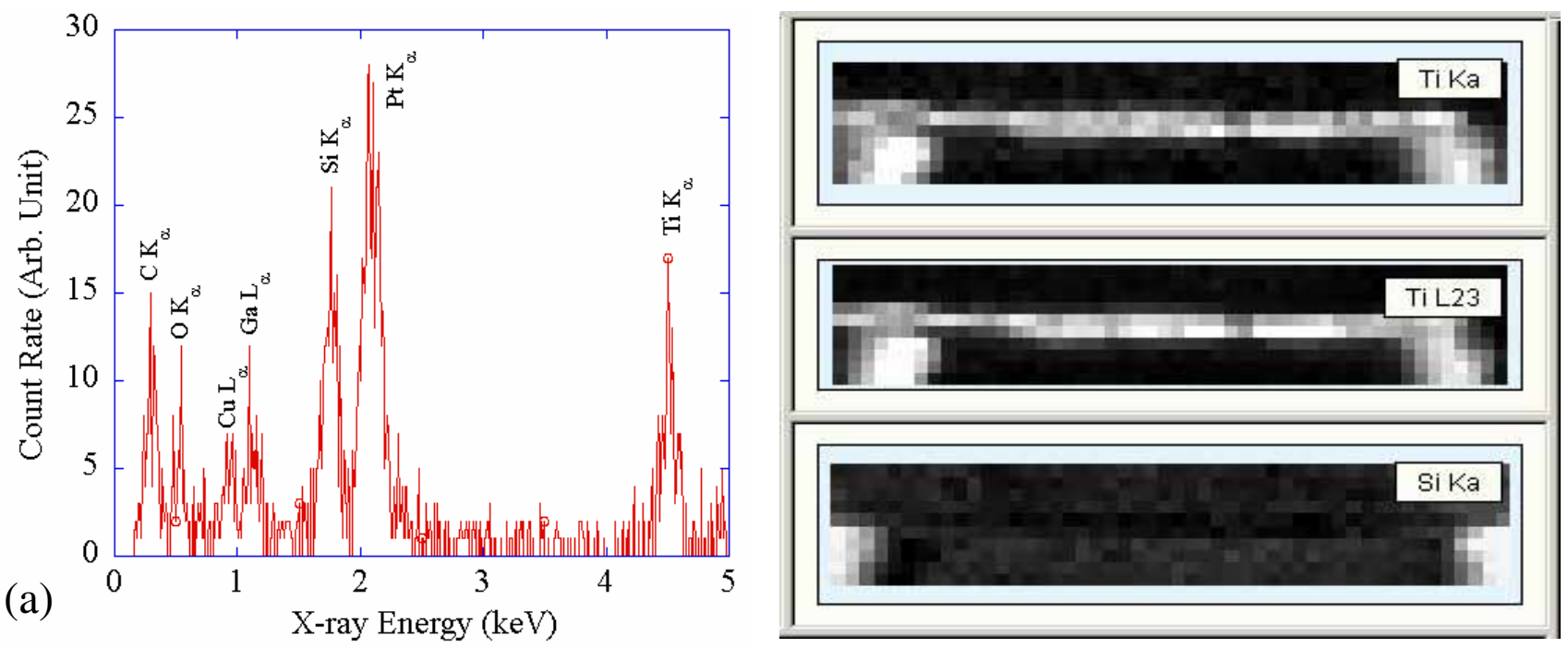

Figure 4. An EDS spectrum acquired from the faint layer between the two W-plugs (a) and elemental maps of Ti based on integrated intensities of the Ti $\mathrm{K}_{\alpha} \mathrm{X}$-ray and the $\mathrm{Ti} \mathrm{L}_{2,3}$ edge, and the Si $\mathrm{K}_{\alpha} \mathrm{X}$-ray.

point. The sample was tilted around an axis that is perpendicular to the layer and toward the EDS detector, by about $10^{\circ}$. This was done to eliminate any possibility of the $\mathrm{Cu}$ mesh blocking X-rays generated from the sample, while the edge-on view of the faint layer was retained.

An EDS spectrum acquired with the nano-probe positioned in the middle of the faint layer is shown in Fig. 4(a). Characteristic X-rays of Ti, Si, O, Cu, Pt, Ga and C are present. Among these, the Cu signals are from background generated from the $\mathrm{Cu}$ mesh, those of $\mathrm{Si}$ and $\mathrm{O}$ have contributions from redeposition of $\mathrm{SiO}_{2}$ and possibly that of some $\mathrm{Si}$ caused by the FIB milling, that of $\mathrm{Pt}$ is from the Pt deposited as a protection coating during FIB, that of Ga from the Ga ions used in FIB, and that of C from the $\mathrm{C}$ supporting film and contamination by the electron beam. The only element that was likely to be from the process is Ti. As a result, Ti elemental mapping was performed based on integrated intensities of the $\mathrm{Ti} \mathrm{K}_{\alpha} \mathrm{X}$-ray and the $\mathrm{Ti} \mathrm{L}_{2,3}$ edge. The resulting elemental maps are presented in Fig. 4(b). The maps reveal that the faint layer contains Ti. The layer is some Ti remaining from the Ti seed deposition used in forming the tungsten-plugs, and was left behind after post-W CMP (chemicalmechanical planarization). Based on this analytical result, the pressure applied on the CMP polishing pads was increased, after which such a bridging problem has not been observed again.

In general, the integrated core-loss intensity alone is not sufficient to reflect the elemental distribution, since it is proportional to not only the areal density of the element, but also the total amount of transmitted electrons, which in turn decreases when the local mean free path of inelastic scattering, $\lambda$, becomes larger. In our case, $\lambda$ for Ti or Ti oxide is larger than that of the surrounding $\mathrm{SiO}_{2}$. Hence the indication of an increased density of $\mathrm{Ti}$ in the faint layer compared with the surrounding $\mathrm{SiO}_{2}$ remains valid even when the $\lambda \mathrm{s}$ of $\mathrm{Ti}$ or $\mathrm{Ti}$ oxide and $\mathrm{SiO}_{2}$ are taken into account.

The PEELS and EDS Ti maps are almost identical between the W-plugs. However, they become significantly different where the $\mathrm{W}$-plugs are. The distribution of $\mathrm{Ti}$ in these two areas becomes broader in the EDS map. However, this is an artifact resulting from the increased background caused by Bremstrahlung due to the presence of the heavy element tungsten. In contrast, the PEELS elemental map is not susceptible to this artifact.

The EDS Si map consistently shows a deficiency of Si along the faint layer. However, the map becomes brightest where the two W-plugs are. This arose actually from the $\mathrm{W}_{\alpha} \mathrm{X}$-ray which is too close to that of $\mathrm{Si} \mathrm{K}_{\alpha}$ to be resolved in EDS. 

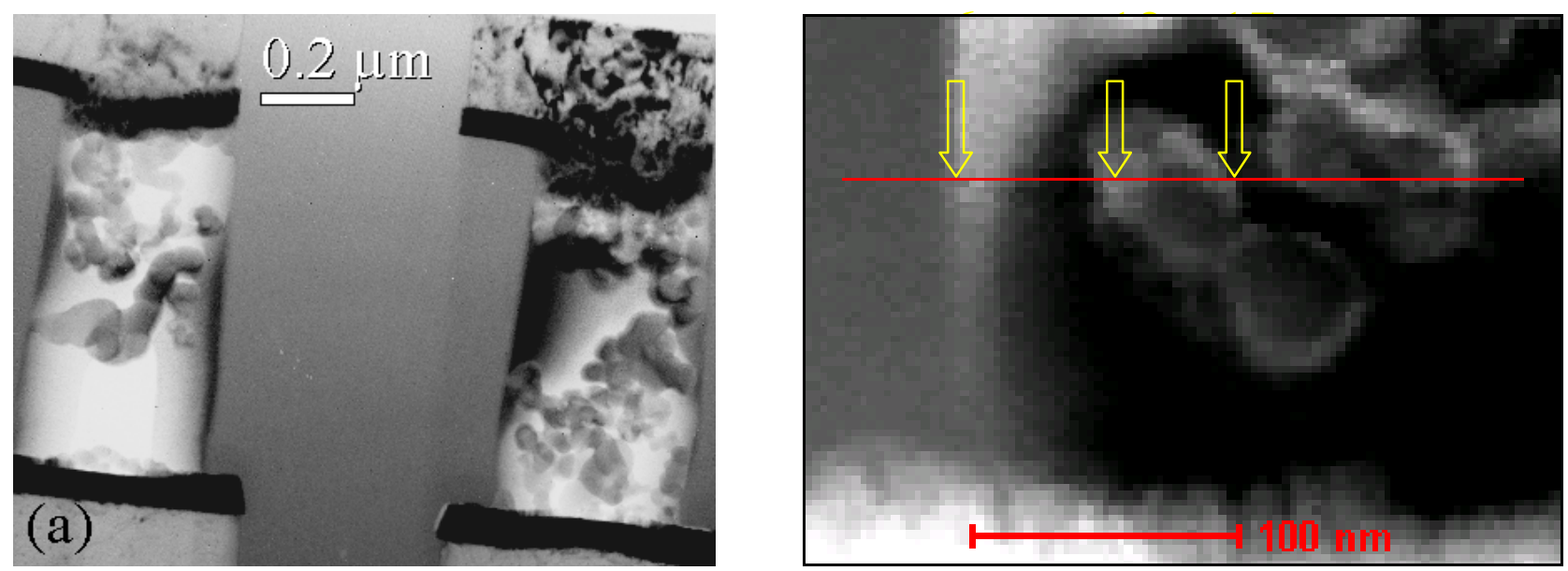

Figure 5. A TEM bright-field image of a pair of vias from a multiple bit failure site where the bits were electrically open (a). The image reveals that there is no metal via-fill, while clusters of particles are present in the vias. Fig. 5(b) is a STEM darkfield image of some particles in the bottom left corner of the via on the right, where EDS-PEELS spectrum profiling was performed. Three positions of the electron probe were labeled along the profile.

EDS and PEELS have also been extremely useful for elemental analysis. The two techniques are complementary in elemental detection efficiency. With the availability of both techniques, the elemental analysis becomes more powerful than either one of them, and especially under the circumstances where extremely high-z and low-z elements are present, such as in the case of hafnia.

Fig. 5(a) is a TEM brightfield image of a pair of vias from a multiple bit failure site where the bits were electrically open. The image reveals that there is no metal via-fill, while clusters of particles are present in the vias. Fig. 5(b) is a STEM darkfield image of some particles (located in the bottom left corner of the via on the right in Fig. 5(a)), where EDS-PEELS spectrum profiling was performed. Three positions of the electron probe along the profile were labeled. The areal density of $\mathrm{C}$ was calculated from the integrated C K edge [8], and the profile is presented in Fig. 6(a) together with those of Si and O obtained from the integrated intensities of their $\mathrm{K}_{\alpha} \mathrm{X}$-rays. The analysis showed a presence of $\mathrm{Si}$ and $\mathrm{O}$ in the particles, and $\mathrm{C}$ between the particles. The $\mathrm{L}_{2,3}$ edge of $\mathrm{Si}$ in $\mathrm{SiO}_{2}$ was observed from the particle between positions 12 and 17, and is shown in Fig. 6(b).

The above observations suggest that after opening of the vias, there was remnant photoresist in the vias. The remnant photoresist prevented the filling of the vias by TiW. In addition, subsequent CMP introduced $\mathrm{SiO}_{2}$ particles from the ILD (interlayer dielectric).

\section{CONCLUSIONS}

This paper presented examples of the application of FIB/SEM and TEM to bit failure analyses in SRAM arrays. Dual-beam FIB was effective in exposing the abnormal features that were responsible for the bit failures. In some analyses, FIB/SEM was sufficient to identify the root causes of the problems. While there are occasions where the FIB/SEM resolution was not sufficient, and TEM/STEM and the associated EDS-PEELS techniques were resorted to. In addition to physical structure information, 

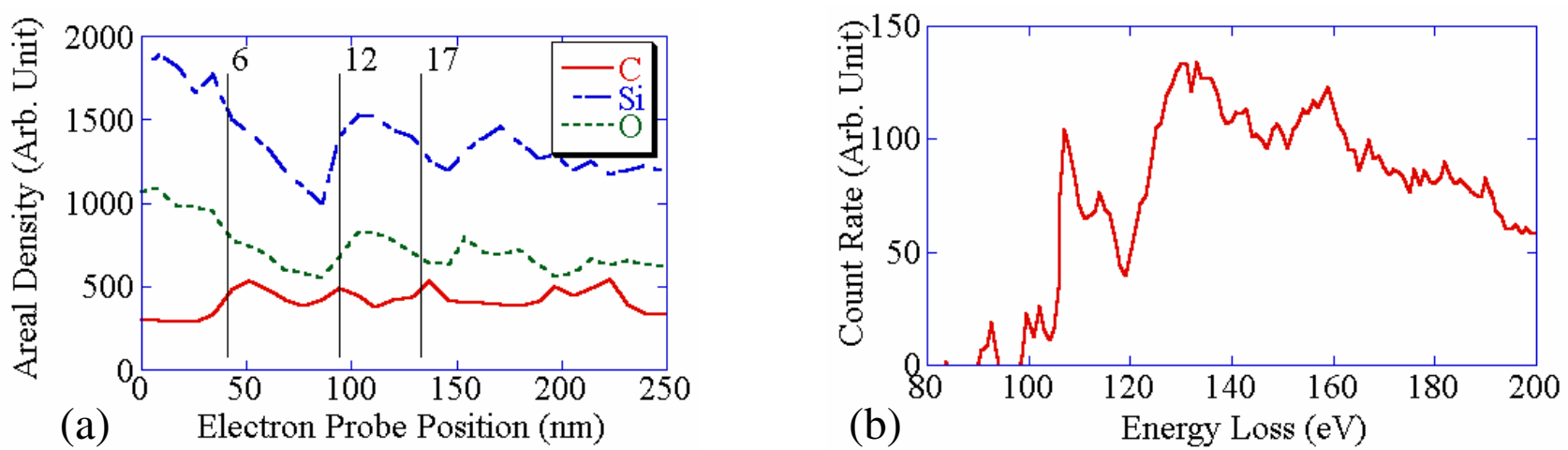

Figure 6. Profiles of the areal densities of $\mathrm{C}$ (acquired from PEELS), Si and $\mathrm{O}$ (obtained from the integrated intensities of their $\mathrm{K}_{\alpha} \mathrm{X}$-rays) (a), and the $\mathrm{L}_{2,3}$ edge of $\mathrm{Si}$ observed from the particle between positions 12 and 17 of the electron probe (b).

elemental distribution and chemical bonding status could be acquired from the features of interest down to the size scale of nanometers. Process issues including open vias, discontinuous metal-lines, metal-line bridging, incomplete CMP were identified.

\section{REFERENCES}

1. Shuji Ikeda, Yasuko Yoshida, Koichiro Ishibashi, and Yasuhiro Mitsui, "Failure Analysis of 6T SRAM on Low-Voltage and High-Frequency Operation", IEEE Trans. Elec. Devices, 50 (5), 1270 (2003).

2. Soon-Moon Jung, Jun-Sup Uom, Won-Suek Cho, Yong-Joon Bae, Yeon-Kyu Chung, KwangSuk Yu, Kil-Yeon Kim, and Kyung-Tae Kim in A Study of Formation and Failure Mechanism of CMP Scratch Induced Defects on ILD in a W-damascene Interconnect SRAM Cell (Proceedings of $39^{\text {th }}$ Annual International Reliability Physics Symposium, Orlando, FL, 2001) p. 42,

3. Z. G. Song, G. Qian, J. Y. Dai, Z. R. Guo, S. K. Loh, C. S. Teh and S. Redkar in Application of Contact-level Ion-beam Induced Passive Voltage Contrast in Failure Analysis of Static Random Access Memory (Proceedings of $8^{\text {th }}$ IPFA, Singapore, 2001) p. 103.

4. C. S. Teh, Z. G. Song, J. Y. Dai, Z. R. Guo and S. Redkar in Poly-residue-induced Contact Failure in 0.18um Technology (Proceedings of $8^{\text {th }}$ IPFA, Singapore, 2001) p. 117.

5. D. W. Susnitzky and K. D. Johnson, Microscopy and Microanalysis, 4, 656 (1998).

6. J. F. Walker and R. F. Broom, Proceedings of Microscopy of Semiconductor Materials Conference, 157, 473 (1997)

7. R. B. Jamison, A. J. Mardinly, D. W. Susnitzky, R. Gronsky, Microscopy and Microanalysis 6, 526 (2000).

8. R. Egerton in Electron Energy-Loss Spectroscopy in the Electron Microscope (Plenum Press, New York and London, 1996), p. 280. 